\title{
Nanocluster structure deduced from AC-STEM images coupled to theoretical modelling
}

\author{
K. Sukuta ${ }^{1}$, M. Van den Bossche ${ }^{1}$, A. Pedersen ${ }^{1}$, H. Jónsson ${ }^{1,2}$ \\ ${ }^{1}$ Science Institute and Faculty of Physical Sciences, University of Iceland, 107 Reykjavík, Iceland \\ ${ }^{2}$ Center for Nonlinear Studies, Los Alamos, NM 87545, USA \\ hj@hi.is
}

DOI 10.17586/2220-8054-2017-8-6-723-731

\begin{abstract}
Determining the atomic structure of nanoclusters is a challenging task and a critical one for understanding their chemical and physical properties. Recently, the high resolution aberration corrected scanning transmission electron microscope (AC-STEM) technique has provided valuable information about such systems, but the analysis of the experimental images has typically been qualitative rather than quantitative. A method is presented for detailed analsis of AC-STEM images combined with theoretical modelling to extract atomic coordinates. An objective function formed by a linear combination of a fit to the two-dimensional AC-STEM image plus an estimate of the cluster's energy for adding information about the third dimension is used in a global optimization algorithm to extract the atomic coordinates. The method is illustrated by analyzing model images generated for the Garzón structure of the $\mathrm{Au}_{55}$ cluster, which is a metastable structure for the embedded atom method (EAM) potential function used here to estimate the total energy. As the method does not rely on the alignment of atom rows in the AC-STEM image, the partially disordered chiral structure of the $A_{55}$ can successfully be determined even when a significant level of noise is added to the images.
\end{abstract}

Keywords: nanocluster, AC-STEM, atomic structure, global optimization, genetic algorithm.

Received: 24 November 2017

Revised: 27 November 2017

\section{Introduction}

Modern chemistry and condensed matter physics is based on knowing the atomic scale structure of the systems under study. Only after identifying the ordering of the atoms can one reliably assess the various properties such as chemical reactivity. The relationship between structure and function underpins all modern chemical intuition and understanding. Heterogeneous catalysis occurs, for example, on the surface of solids and for a long time a serious hindrance in understanding the catalytic activity was the difficulty in determining the atomic scale structure of solid surfaces. While it is relatively easy to determine atomic ordering in bulk crystals with X-ray scattering, the surface layer of atoms, which can have a significantly different atomic composition and structure than the bulk, gives a too small signal to be 'visible'. With the advent of the scanning tunneling microscope in the early 1980's [1], this problem was largely solved, and since then, there has been an explosion in the study of structure and chemical reactivity of surfaces and a great leap forward in understanding heterogeneous catalysis.

Nanoscale clusters of atoms have shown remarkable catalytic activity. With modern nanotechnology, it is possible to prepare such clusters and deposit them in a non-destructive manner on solid surfaces or into porous solids. It has, for example, been shown that $\mathrm{CO}$ oxidation, an important reaction for cleaning exhaust, can occur readily at below room temperature in the presence of $\mathrm{Au}$ nanoclusters deposited on a $\mathrm{TiO}_{2}$ surface if the diameter of the clusters is in the range of $2-3 \mathrm{~nm}$, corresponding to ca. $10^{3}$ atoms. For larger as well as smaller clusters, the catalytic activity per site drops dramatically [2]. This and similar observation on other nanocluster catalysts remain puzzling. A consensus on the reason for the strong size dependence of the catalytic activity has not been reached. In other experiments, the catalytic activity of transition metal nanoclusters has been found to depend strongly on preparation conditions and slight variations in composition [3].

A major obstacle for gaining a better understanding of nanocluster catalysis is the lack of information about the atomic structure of the clusters. While, for example, gold crystallizes in the face centered cubic (FCC) structure, small $\mathrm{Au}$ clusters can have very different atomic ordering by forming, for example, icosahedral or decahedral structures with fivefold symmetry (see, for example, Ref. [4]) Various theoretical calculations have addressed this problem, using mainly empirical potential functions to describe the interaction between the Au atoms, electronic density functional theory (DFT), or a combinations of both. But, it is extremely hard to search for the optimal structure of such a cluster even if it contains only ca. $10^{2}$ atoms. Also, it is not clear whether the current approaches to describing the atomic interactions are sufficiently accurate for these unusual systems. 
An experimental technique that can provide information about the atomic scale structure of nanoclusters deposited on surfaces has been lacking until the recent development of the aberration-corrected scanning transmission electron microscope (AC-STEM). Based on ideas presented in the late 1990's [5,6], the first such instrument was built in Daresbury in England in 2001 and several such instruments have now been set up at major universities and research laboratories. Publications describing structural determination of nanoclusters and other nanosized systems using AC-STEM have appeared in the past decade, see for example Refs. [7-12].

While the AC-STEM experimental equipment and the refinements of the measured images are highly developed, the analysis of the images in terms of atomic structure of the clusters is still often qualitative. In some cases, a catalog of calculated images based on ideal cluster structures is generated and the measured images then compared to the catalog by eye. This type of analysis is in many cases good enough to determine, for example, which one of the three main categories of structures a measured metallic cluster belongs to: FCC, icosahedra and decahedra. However, more information could be extracted from the images with a quantitative analysis tool. For example, in order to understand catalytic properties, it is important to obtain information about the atomic ordering at the surface of the cluster. The atoms at the surface of a cluster may order in a different way than would be expected from the ordering in the interior, as is well known for the surfaces of crystals.

In this article, we describe an algorithm that can be used to extract atomic coordinates from an AC-STEM image. The method is based on a two-step procedure. First, atomic coordinates in the plane of the AC-STEM image are generated. Then, the image is complemented with an estimate of the energy of the cluster to determine the three-dimensional structure. The algorithm is applied to an analysis of the $\mathrm{Au}_{55}$ cluster. This is an interesting test case for two reasons. Firstly, Garzón and coworkers have carried out extensive studies of this cluster using the Gupta potential and shown that the structure is chiral while being highly disordered [13-15] (see Fig. 2). We will refer to this as the Garzón structure of $\mathrm{Au}_{55}$. Experimental AC-STEM studies have supported this prediction as ca. half of the measured images were found to be consistent with the Garzón structure [16]. The other half of the images were ascribed to higher energy configurations due to thermal energy and electron beam interaction with the clusters but the atomic coordinates of those configurations were not determined. No high symmetry structures were observed. The analysis was based on image simulations using multiple scattering calculations [16].

We choose the $\mathrm{Au}_{55}$ cluster as a demonstration for our method because it is more challenging than a structure where the atoms line up in rows. Furthermore, it is small enough that the globally optimal structure can be found without too much computational effort. Recently, a method for extracting atomic coordinates from AC-STEM images was presented, but it relies on the atoms forming rows and cannot be applied to structures such as the Garzón structure [17]. The estimate of the energy of the cluster is obtained from the embedded atom method (EAM) potential energy function [18] rather than the Gupta potential because EAM predicts a slightly different optimal structure than the Garzón structure. This choice reflects the fact that the energy of a cluster can rarely be calculated to high precision, whether the calculation is based on an empirical potential or DFT. But, when combined with an AC-STEM image the correct structure should be obtained despite errors in the energy. Fig. 1 shows a comparison of the Garzón structure and the optimal structure predicted by the EAM potential. The average difference in atomic coordinates is $0.52 \AA$. The Garzón structure is metastable for the EAM potential function and is $0.1 \mathrm{eV}$ higher in energy than the global minimum on the EAM energy surface. Nevertheless, the method presented here for analysis of AC-STEM images is able to converge on the atomic coordinates of the Garzón structure even though it relies on an EAM energy estimate.

\section{Methodology}

The method for extracting atomic coordinates from an AC-STEM image in combination with an estimate of the cluster energy is described in this section. Instead of analyzing an experimental AC-STEM image at this stage, we use for testing and presentation purposes a simulated AC-STEM image for the Garzón structure of the $\mathrm{Au}_{55}$ cluster. We will refer to this as the target image and it is specified by the intensity of pixels in the $x-y$ plane, $I_{\text {tar }}(x, y)$. Following Li et al., we assume each of the $N=55$ atoms is the source of a signal described by a Gaussian [7]:

$$
I_{\text {tar }}(x, y)=\sum_{i=1}^{N} \exp \left(\frac{-\left(x-x_{i}\right)^{2}+\left(y-y_{i}\right)^{2}}{2 \sigma^{2}}\right)
$$

where $x_{i}, y_{i}$ denotes coordinates of atom $i$. The width of the Gaussian, $\sigma$, is chosen to have the value $0.78 \AA$ as was deduced by Li et al. from their experimental AC-STEM data on Au nanoclusters [7]. A similar value, $0.8 \AA$, was deduced by He et al. [19]. 


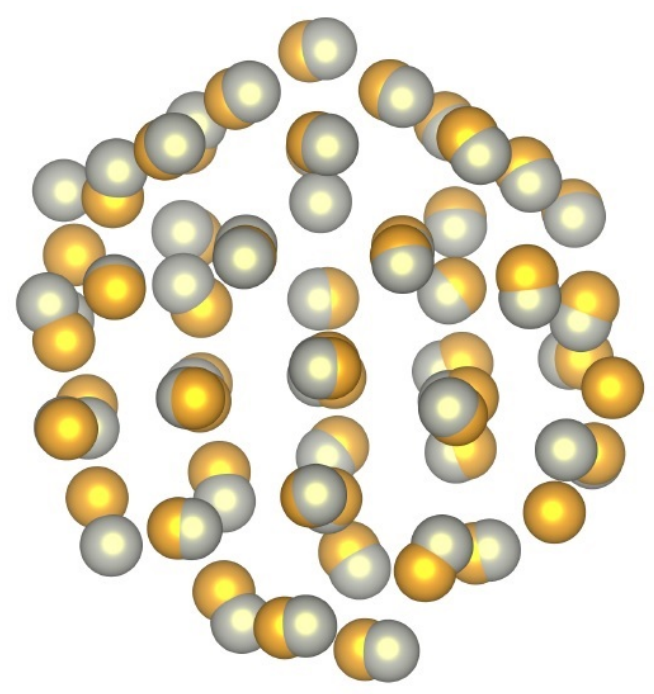

FIG. 1. Comparison of the Garzón structure (the global minimum on the Gupta energy surface) and the EAM optimal structure (the global minimum on the EAM energy surface). The Garzón structure has $0.1 \mathrm{eV}$ higher energy than the EAM optimal structure when the energy is evaluated with the EAM potential. The average difference in the distance between corresponding atoms in the two structures is $0.52 \AA$.
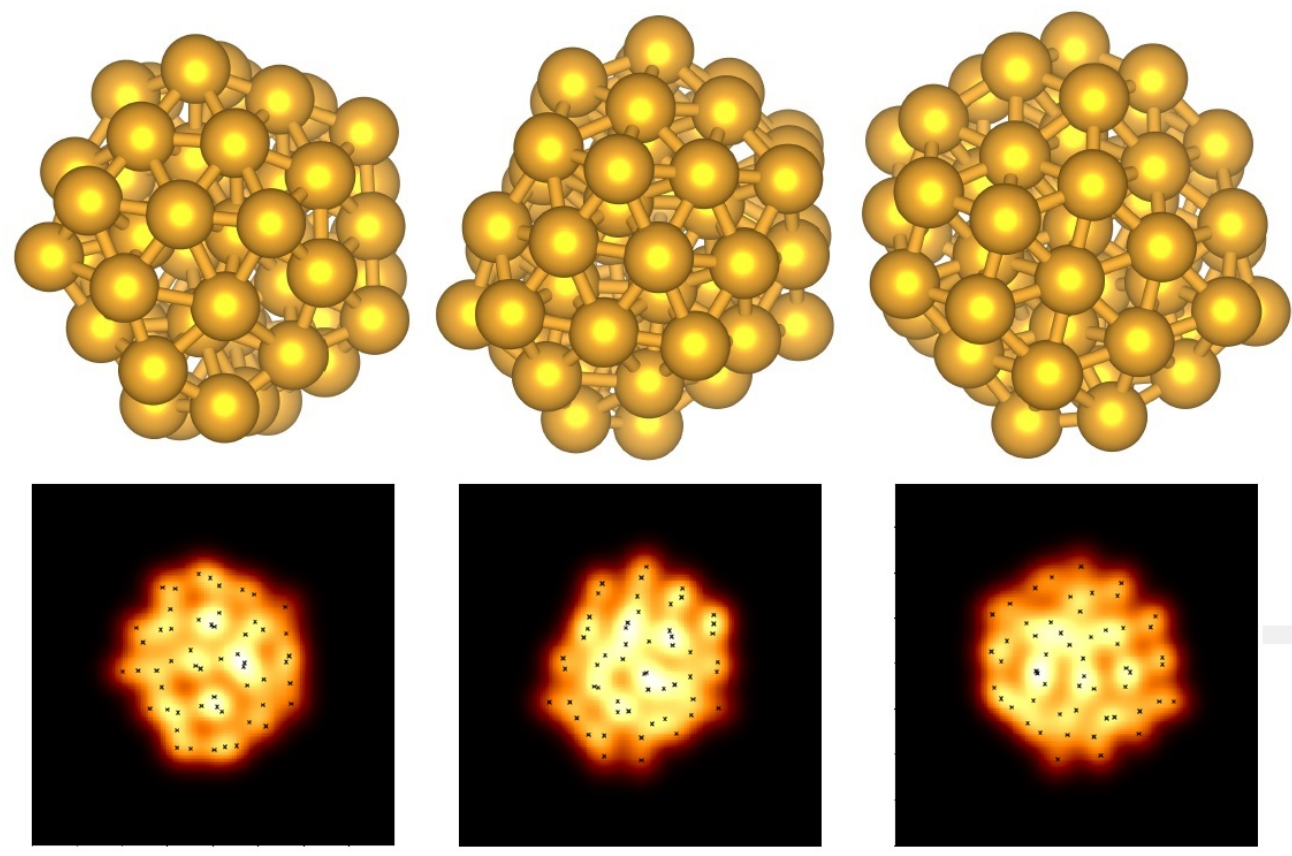

FIG. 2. Three different views of the Garzón structure of a $\mathrm{Au}_{55}$ cluster (top), referred to as viewing angle \#1, \#2 and \#3, and simulated AC-STEM images (bottom). The STEM images cover an area of $20 \AA \times 20 \AA$ and the atomic positions are indicated as black crosses.

Simulated AC-STEM images for three different viewing angles are shown in Fig. 2. Various levels of noise are also added to the images to better emulate experimental data, as described below. The target image is normalized by summing the intensity of all pixels in the image and then dividing the intensity of each pixel with the sum.

The method for generating atomic coordinates from a target image consists of two stages. First, coordinates of the atoms in the plane of the image are generated. This will be referred to as the $x-y$ plane. Then, several threedimensional structures are created by generating $z$-coordinates for the atoms and a GA optimization is performed to minimize an objective function constructed from a weighted sum of the difference between target and simulated 
images plus an estimate of the potential energy of the cluster. The procedure should, in general, be repeated for different choices of the total number of atoms to find the value that gives best agreement with the target image. The procedure is described in detail in the following paragraphs.

\subsection{Generation of $x-y$ coordinates}

The first step is to determine $(x, y)$ coordinates for the atoms in such a way that a simulation image, $I_{\text {sim }}$, best fits a target image, $I_{t a r}$ (in practice an experimentally measured AC-STEM image, but here the target image is calculated from the Garzón structure in order to have a well-defined test problem).

Each atom, $i$, located at $\left(x_{i}, y_{i}\right)$, makes a contribution to the simulated intensity at a pixel $(x, y)$ given by:

$$
I_{a}\left(x, y, x_{i}, y_{i}\right)=\frac{1}{2 N \pi \sigma^{2}} \exp \left(\frac{-\left(x-x_{i}\right)^{2}+\left(y-y_{i}\right)^{2}}{2 \sigma^{2}}\right) .
$$

The normalization is chosen in such a way that the total intensity of $\mathrm{N}$ atoms matches the total intensity of the target image. A reasonable estimate for the peak width $\sigma$ is required. This can be obtained by fitting the width of an isolated feature that is likely to correspond to an isolated atom, or by fitting to the width of a brighter spot corresponding to a column of atoms, if present. Atoms are assigned $(x, y)$ coordinates in the following way. The pixel with highest intensity is selected and the first atom placed at this location in the $x-y$ plane. Then, the contribution of this atom is subtracted from the target intensity. The second atom is placed at the maximum of the reduced target intensity, $I_{\text {tar }}(x, y)-I_{a}\left(x, y, x_{1}, y_{1}\right)$, etc.

After $(x, y)$ coordinates have been assigned to all $N$ atoms, they are refined by minimizing the difference between the simulated and target images:

$$
\begin{aligned}
S_{\text {image }} & =\sum_{\text {pixels } p} D\left(x_{p}, y_{p}\right)^{2}, \\
D\left(x_{p}, y_{p}\right) & =\left(\sum_{i=1}^{N} I_{a}\left(x_{p}, y_{p}, x_{i}, y_{i}\right)\right)-I_{\operatorname{tar}}\left(x_{p}, y_{p}\right) .
\end{aligned}
$$

$S_{\text {image }}$ represents the sum over all pixels of the difference squared between the simulated and target images. The steepest descent displacement vector for each atom that reduces the discrepancy most rapidly is obtained by differentiating $S_{\text {image }}$ with respect to the atomic coordinates. For example, the $x$-component of the steepest descent vector for atom $i$ is:

$$
\begin{aligned}
\frac{\partial S_{\text {image }}}{\partial x_{i}} & =\sum_{\text {pixels } p} 2 D\left(x_{p}, y_{p}\right) \frac{\partial D\left(x_{p}, y_{p}\right)}{\partial x_{i}} \\
& =\sum_{\text {pixels } p} 2 D\left(x_{p}, y_{p}\right) \frac{\partial I_{a}\left(x_{p}, y_{p}, x_{i}, y_{i}\right)}{\partial x_{i}} \\
& =\sum_{\text {pixels } p} 2 D\left(x_{p}, y_{p}\right) I_{a}\left(x_{p}, y_{p}, x_{i}, y_{i}\right)\left(\frac{x_{i}-x_{p}}{\sigma^{2}}\right) .
\end{aligned}
$$

A similar expression applies to the $y$-components of the coordinates of the atoms.

\subsection{Addition of $z$-coordinates}

After $(x, y)$ coordinates have been assigned, the task is to generate a best estimate of the $z$-coordinates. As an initial estimate, $z$ coordinates are randomly chosen in such a way that the cluster is roughly spherical and no two atoms are too close together. An improved estimate is then generated by defining an objective function that includes the energy of the cluster, $E_{\text {pot }}$, plus the difference in simulated and target images. Here, an EAM potential function constructed by Foiles et al. [18] is used to estimate the energy of the cluster, even though it is known not to have the Garzón structure as the global minimum on the energy surface. The combined objective function, $Z$, is:

$$
Z\left(r_{1}, r_{2}, \ldots, r_{N}\right)=E_{\text {pot }}\left(r_{1}, r_{2}, \ldots, r_{N}\right)+\chi S_{\text {image }}\left(r_{1}, r_{2}, \ldots, r_{N}\right) .
$$

The weighting factor $\chi$ is required to bring the potential energy and the image difference on a similar scale. We found it advantageous to start the local minimizations with $\chi=0$ and to gradually increase this value to $\chi=$ $100 \mathrm{eV}$ over the course of the first 50 steps in the local minimization loop.

The potential energy $E_{\text {pot }}$ of the nanocluster is estimated using the LAMMPS software [20]. This estimate does not need to be highly accurate, the most important information is in the image, but without some estimate of the optimal distance between atoms it is not possible to assign the $z$-coordinates. The gradient of the total objective 


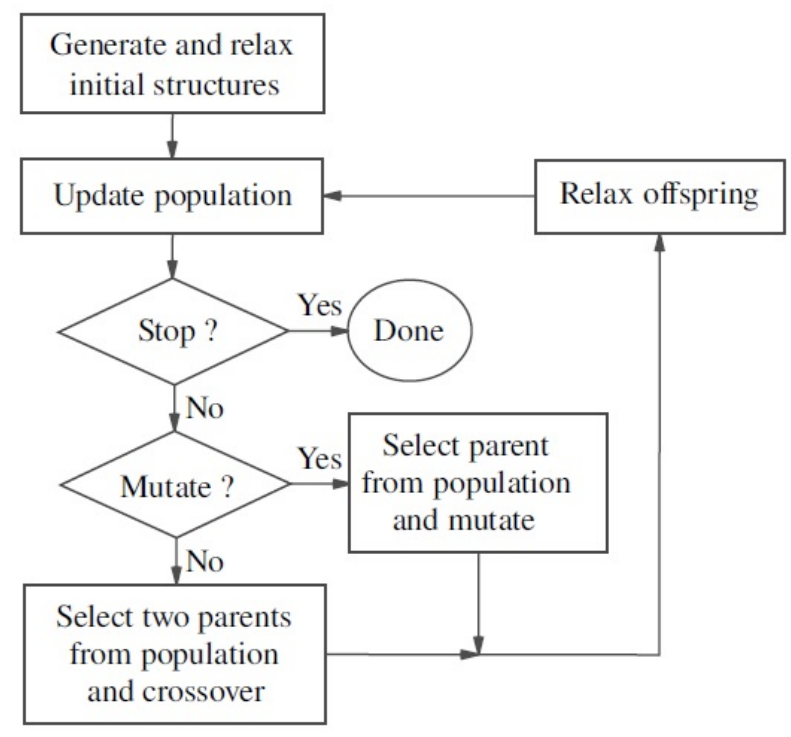

FIG. 3. Flowchart of the genetic algorithm used after a population of cluster structures consistent with the AC-STEM image have been generated.

function then consist of the negative EAM atomic force plus a $\chi$-weighted contribution from the difference in the images, $S_{\text {image }}$.

The full set of coordinates of the atoms in the cluster is refined by local minimization of the objective function (Eq. 8) using the BFGS algorithm [21].

\subsection{Genetic algorithm optimization}

A global optimization of the atomic coordinates is then carried out by minimizing the objective function, Eqn. (8), using genetic algorithm (GA) tools [22] implemented in the Atomic Simulation Environment (ASE) $[23,24]$. The outline of the GA used here is shown in Fig. 3. Twenty initial structures are generated as described in the previous subsection. They all have the same $x$ - and $y$-coordinates for the atoms and the simulated image reproduces well the target image, but they differ in the $z$-coordinates.

The GA loop starts from a population of lowest energy structures that are also sufficiently distinct, as judged from fingerprint functions (see [25]). New structures are generated either by crossover or mutation (a $25 \%$ mutation probability was used). The required number of parent structures are drawn from the population with a bias towards the more stable and less frequently selected structures (as in Ref. [26]). In the case of crossover, the offspring is produced through standard cut-and-splice pairing of two parent structures [27]. Single-parent mutation can occur in three different ways, as listed in Table 1 . The energy of the resulting structure is subsequently minimized using the BFGS algorithm until the largest atomic force drops below $10^{-2} \mathrm{eV} / \AA$. The minimized structure is then added to the list of candidate structures.

\section{Results}

Figure 4 shows the convergence behavior of the GA calculations for the three orientations of the Garzón structure. In all three cases, the Garzón structure is found with near $100 \%$ success rate within 200 GA steps. On

TABLE 1. The various mutation schemes used in the GA optimization.

\begin{tabular}{lcl}
\hline \hline Mutation & Probability & Description \\
\hline Rattle & $60 \%$ & $\begin{array}{l}\text { Randomly displace the atoms with amplitudes } \\
\text { uniformly sampled between } 0 \text { and } 1.5 \AA . \\
\text { Match }\end{array} 30 \%$ \\
Force the atomic positions to the initial $(x, y)$ \\
data set after matching using the Hungarian algorithm $[28,29]$. \\
Compress the structure along the $z$ direction.
\end{tabular}




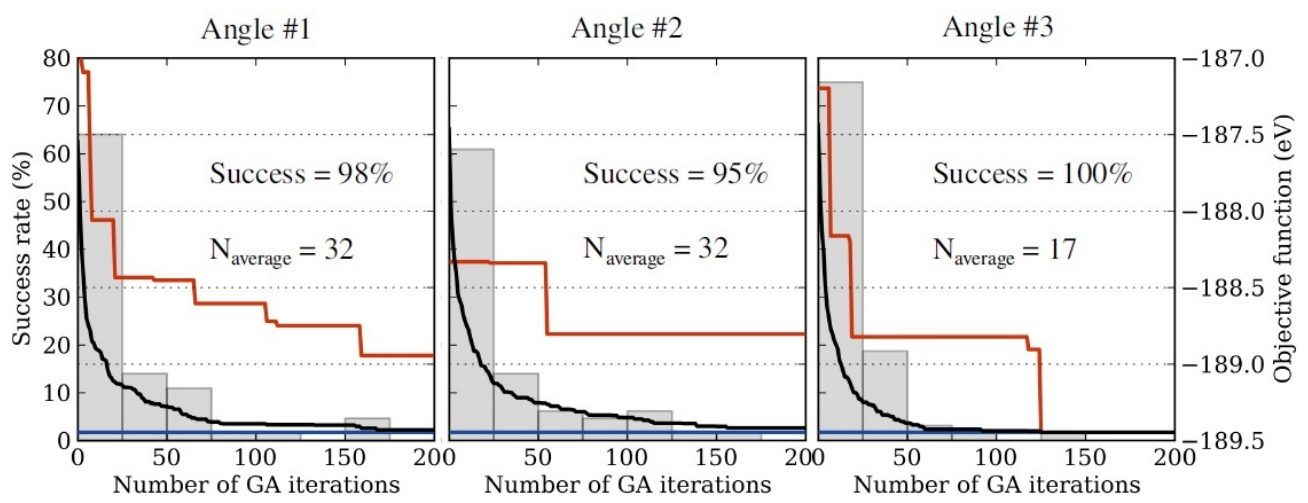

FIG. 4. Hartke graphs of the GA convergence for the three different viewing angles. The statistics are gathered on the basis of 64 independent GA runs. The gray histograms show the distribution of the required number of steps before the Garzón structure is found. The lines represent the evolution of the best-scoring candidating in the best performing (lowest curve) and worst performing (highest curve) runs and the average over the 64 runs (middle curve).

average, circa 30 GA steps are required. A small fraction of the GA runs already finds the GM within the initial population obtained after the first step and random generation of $z$-coordinates for a near spherical cluster. Such AC-STEM-assisted GA runs tend to converge at a faster rate than regular GA runs without the first step involving only the AC-STEM image fitting. When only the potential energy is used as the objective function, we find the success rate to be only $40 \%$ after 1000 iterations, requiring circa 470 steps on average to find the global minimum on the EAM surface.

Noise is added to the target AC-STEM image by adding random numbers drawn from a Gaussian distribution $G$ centered at zero and with a standard deviation proportional to the local intensity:

$$
I_{n o i s-t a r}(x, y)=I_{t a r}(x, y)+\eta
$$

where $\eta$ is a Gaussian random variable obtained from the probability distribution given by

$$
G\left(\eta, \sigma_{n}\right)=\frac{1}{\sqrt{2 \pi \sigma_{n}^{2}}} e^{-\eta^{2} / 2 \sigma_{n}^{2}}
$$

where $\sigma_{n}=\beta I_{\text {tar }}(x, y)$.

Three different noise levels were used setting the proportionality constant in the width of the Gaussian, $\beta$, to 0.1, 0.2 and 0.5, respectively. The resulting images are shown in Fig. 5. At the highest noise level, $\beta=0.5$, some pixels acquire negative intensities. The added noise is then adequately reduced by subsequent Wiener filtering [30]. The images obtained after such filtering are also shown in Fig. 5. as well as convergence graphs for GA calculations employing the filtered images. When comparing to the data in Fig. 4, it can be concluded that using the noisy but filtered images in the GA optimization only slightly increases the number of steps required to reach convergence. The bottom panel in Fig. 5 confirms that the GM structures obtained using the filtered images agree closely with the reference Garzón structure. This indicates that the method is robust with respect to the presence of noise in the AC-STEM images.

We have also performed tests where the target image is generated from a structure that has been perturbed from the Garzón structure by adding random displacements in the $x$ and $y$ positions, drawn from a uniform distribution $[0, \alpha[$. Such tests effectively correspond to evaluating the relative difficulty of finding an optimal structure which is not close to a local minimum structure of the potential energy surface. The convergence data obtained for maximal displacements of $\alpha=0.2 \AA, 0.5 \AA$ and $1.0 \AA$ are shown in Fig. 6. It can be noted that the convergence speed is reduced, but that fair success rates are still achieved even at the large maximal displacement of $1.0 \AA$. The corresponding atomic displacements and changes in the simulated AC-STEM image are illustrated in Fig. 6. We conclude from these tests that it is advisable (though not critically important) to use sufficiently accurate representations of the potential energy surface, such that the real structure is not too distant from a local minimum on the potential energy surface. 


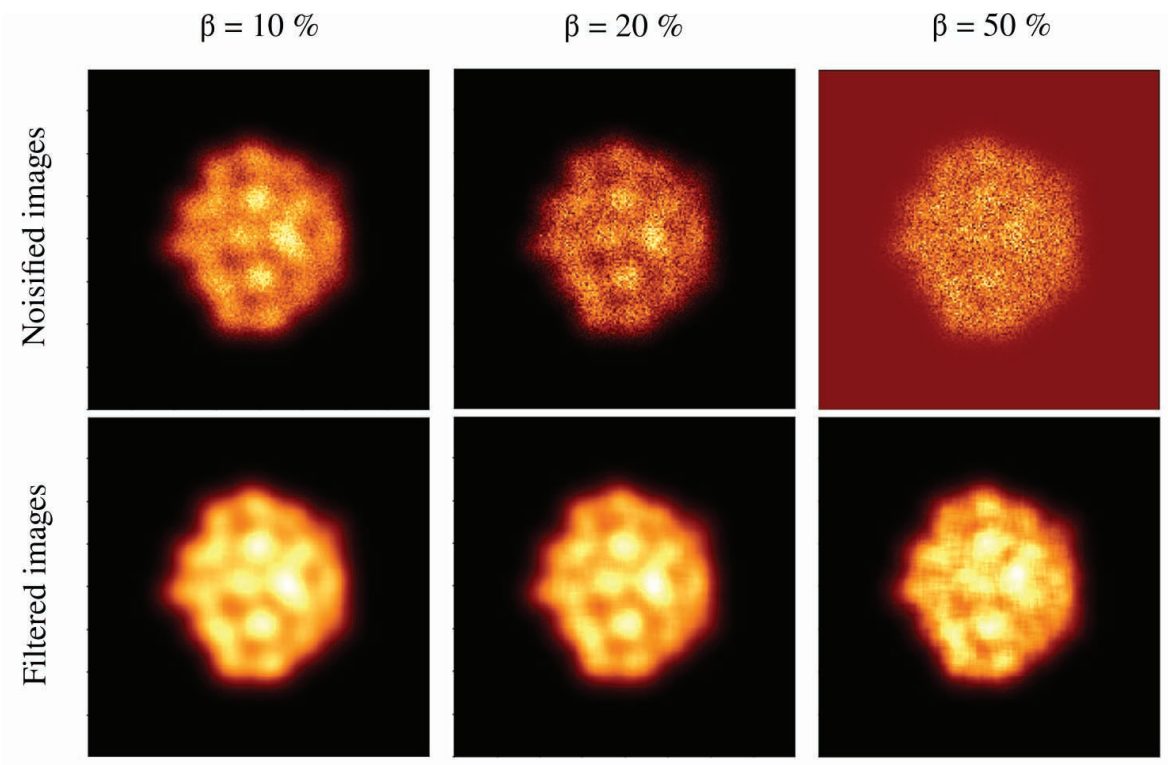

Hartke plots of the GA convergence gathered from 64 independent runs

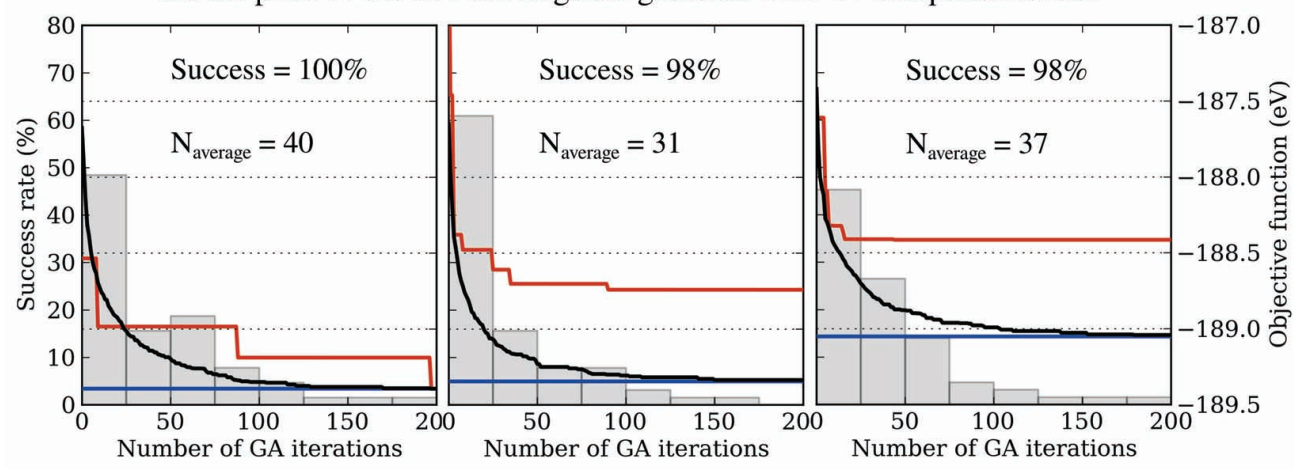

Comparison of the GM structure obtained with (๑) and without (๑) noise
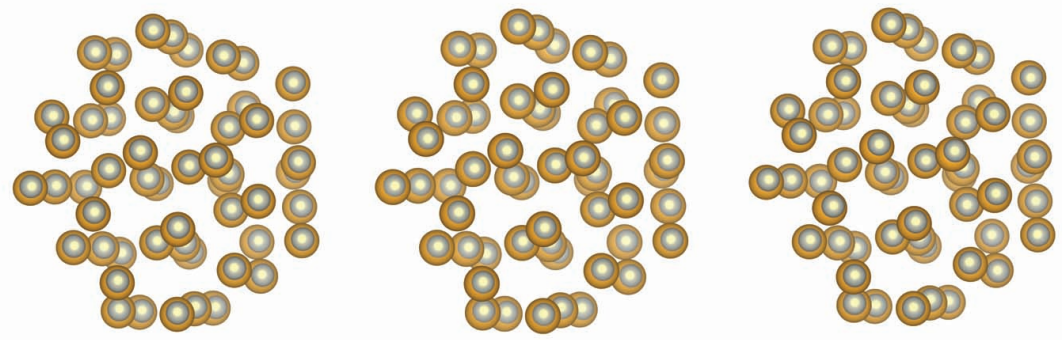

FIG. 5. Top: simulated STEM images with added noise levels (top row) and the corresponding images after Wiener filtering (second row). Center: Hartke graphs showing the convergence of the corresponding GA calculations (bars and lines as in Fig. 4). Bottom: 2-dimensional projections of the Garzón structure (gold) and of the results of the GA calculations with the various noise levels (gray). The latter have been displaced towards the viewer by $0.8 \AA$ to make it easier to compare the positions in the $x-y$ plane.

\section{Discussion}

The method presented here for the extraction of atomic coordinates from AC-STEM images supplemented by a method for estimating the cluster energy does not rely on the presence of distinct atomic rows in the ACSTEM image. This is in contrast to the approach by Yu et al. [17], where such rows are employed to create the initial population and to apply specific mutations. A second major difference is that we consistently use the total 

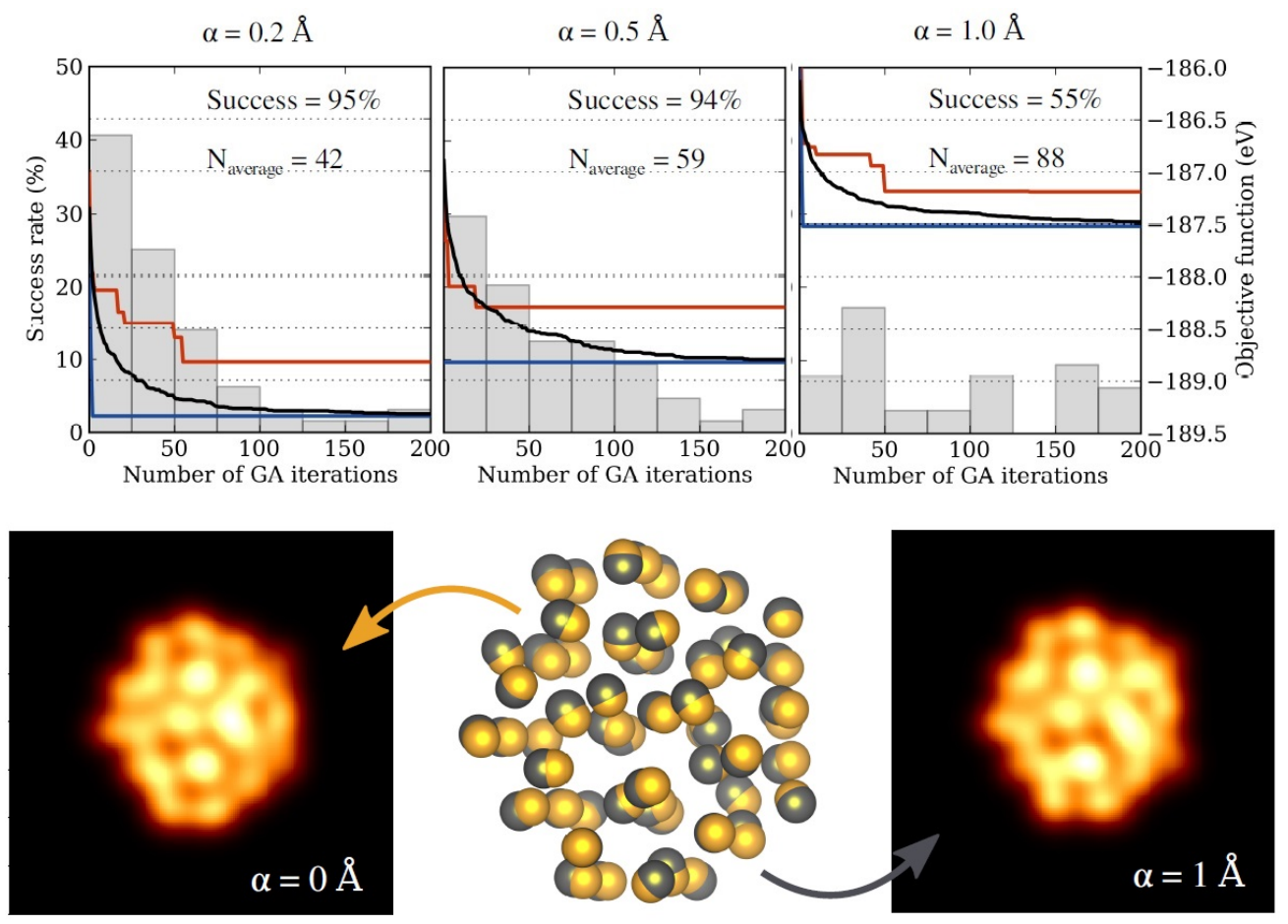

FIG. 6. Top panel: Hartke graphs showing the GA convergence for the three different maximal perturbations from the Garzón structure, $\alpha$, in the generation of the target image. The histogram and lines are of the same kind as in Fig. 4. Bottom panel, center: Overlay of the structural models of the unperturbed (lighter coloured) and the most strongly perturbed structure $(\alpha=1.0 \AA)$ (darker coloured). The atomic radii are $1.5 \AA$, for comparison. Bottom panel, left and right: simulated AC-STEM images for the unperturbed Garzón structure and most strongly perturbed structure.

objective function (Eq. 8) throughout the GA run. Yu and coworkers minimize the potential energy during the local optimization and add the image discrepancy term only as a single-point correction after the optimization.

The tests presented here show that it is possible to successfully use an objective function including both the potential energy and an image discrepancy term, even when the experimental AC-STEM image is affected by large noise. The method can be applied to studies of disordered clusters. It should also be able to identify whether the atoms at the surface of the cluster are ordered in a different way than the atoms in the interior, an important aspect for the study of catalytic properties.

\section{Acknowledgements}

We thank Ignacio Garzón for providing us with the atomic coordinates in the optimal $\mathrm{Au}_{55}$ structure deduced from the Gupta potential function. This work was supported by the Icelandic Research Fund and the University of Iceland Doctoral Fund. KS and MVDB gratefully acknowledge computer resources at the University of Iceland Computing Services (Reiknistofnun) and through a SNIC grant at C3SE (Göteborg, Sweden).

\section{References}

[1] Binning G., Rohrer H., Gerber Ch., Weibel E. Surface studies by scanning tunneling microscopy. Phys. Rev. Letters, 1982, 49(1), P. 57-61.

[2] Valden M., Lai X., Goodman D.W. Onset of catalytic activity of gold clusters on titania with the appearance of nonmetallic properties. Science, 1998, 281, P. 1647.

[3] Yamauchi M., Abe R., Tsukuda T., Kato K., Takata M. Highly selective ammonia synthesis from nitrate with photocatalytically generated hydrogen on $\mathrm{CuPd} / \mathrm{TiO}_{2}$. J. Am. Chem. Soc., 2011, 133(5), P. 1150-1152.

[4] Li H., Li L., Pedersen A., Gao Y., Khetrapal N., Jónsson H., Zeng X.C. Magic-number gold nanoclusters with diameter 1 to 3.5 nm: Relative stability and catalytic activity for CO oxidation. Nano Letters, 2015, 15, P. 682.

[5] Brown L.M. A Synchrotron in a Microscope. Institute of Physics Conference Series, 1997, 153, P. 22.

[6] Krivanek O.L., Dellby N., Lupini A.R. Towards sub-Å electron beams. Ultramicroscopy, 1999, 78(1-4), P. 1-11.

[7] Li Z.Y. al. Three-dimensional atomic-scale structure of size-selected gold nanoclusters. Nature, 2007, 451(7174), P. 46-48.

[8] W Zhou W., Ross-Medgaarden E.I., Knowles W.V., Wong M. S., Wachs I.E., Kiely C.J., Nature Chemistry, 2009,1 , P. 722. 
[9] Van Aert S., Batenburg K.J., Rossell M.D., Erni R., Van Tendeloo G. Three-dimensional atomic imaging of crystalline nanoparticles. Nature, 2011, 470, P. 374

[10] Bals S., Van Aert S., Romero C.P., Lauwaet K., Van Bael M.J., Schoeters B., Partoens B., Yücelen E., Lievens P., Van Tendeloo G., Atomic scale dynamics of ultrasmall germanium clusters. Nature Communications, 2012, 3, P. 897.

[11] Liu J., Single atom and cluster catalysis: The era of aberration-corrected scanning transmission electron microscopy. Microscopy and Microanalysis, 2013, 19 (Suppl. 2), P. 1678.

[12] Corma A. et al., Exceptional oxidation activity with size-controlled supported gold clusters of low atomicity. Nature Chemistry, 2013, 5, P. 775 .

[13] Garzón I.L., Posada-Amarillas A. Structural and vibrational analysis of amorphous Au 55 clusters. Phys. Rev. B, 1996, 54, P. 11796-11802.

[14] Garzón I.L. et al. Lowest Energy Structures of Gold Nanoclusters. Phys. Rev. Letters, 1998, 81, P. 1600-1603.

[15] Michaelian K., Rendón N., Garzón I.L. Structure and energetics of Ni, Ag, and Au nanoclusters. Phys. Rev. B, 1999, 60(3), P. $2000-2010$.

[16] Wang Z.W., Palmer R.E. Experimental evidence for fluctuating, chiral-type Au 55 clusters by direct atomic imaging. Nanoletters, 2012, 12, P. 5510-5514.

[17] Yu M., Yankovich A.B., Kaczmarowski A., Morgan D., Voyles P.M. Integrated computational and experimental structure refinement for nanoparticles. ACS Nano, 2016, 10(4), P. 4031-4038.

[18] Foiles S.M., Baskes M.I., Daw M.S. Embedded-atom-method functions for the fcc metals $\mathrm{Cu}, \mathrm{Ag}, \mathrm{Au}, \mathrm{Ni}, \mathrm{Pd}, \mathrm{Pt}$, and their alloys. Phys. Rev. B, 1986, 33(12), P. 7983-7991.

[19] He D.S., Li Z.Y., Yuan J. Kinematic HAADF-STEM image simulation of small nanoparticles. Micron, 2015, 74(C), P. 47-53.

[20] Plimpton S. Fast parallel algorithms for short-range molecular dynamics. J. Comput. Phys., 1995, 117(1), P. 1-19.

[21] Head J.D., Zerner M.C. A Broyden-Fletcher-Goldfarb-Shanno optimization procedure for molecular geometries. Chem. Phys. Lett., 1985, 122(3), P. 264-270.

[22] Vilhelmsen L.B., Hammer B. Systematic study of $A u_{6}$ to $A u_{12}$ gold clusters on $\mathrm{MgO}(100) \mathrm{F}$ centers using density-functional theory. Phys. Rev. Lett., 2012, 108(12), P. 126101(4 pp.).

[23] Bahn S.R., Jacobsen K.W. An object-oriented scripting interface to a legacy electronic structure code. Comput. Sci. Eng., 2002, 4(3), P. $56-66$.

[24] Larsen A.H., et al. The atomic simulation environment a Python library for working with atoms. J. Phys.: Condens. Matter, 2017, 29(27), P. 273002.

[25] Lyakhov A.O., Oganov A.R., Stokes H.T., Zhu Q. New developments in evolutionary structure prediction algorithm USPEX. Comput. Phys. Commun., 2013, 184(4), P. 1172-1182.

[26] Vilhelmsen L.B., Walton K.S., and Sholl D.S., Structure and mobility of metal clusters in MOFs: Au, Pd, and AuPd clusters in MOF-74. J. Am. Chem. Soc., 2012, 134(30), P. 12807-12816.

[27] Daven D.M., Tit N., Morris J. R., Ho K.M. Structural optimization of Lennard-Jones clusters by a genetic algorithm. Chem. Phys. Lett., 1996, 256(12), P. 195-200.

[28] Kuhn H.W. The Hungarian method for the assignment problem. Naval Research Logistics, 1955, 2(1-2), P. 83-97.

[29] Munkres J. Algorithms for the assignment and transportation problems. J. Soc. Ind. Appl. Math., 1957, 5(1), P. 32-38.

[30] Kilaas R. Optimal and near-optimal filters in high-resolution electron microscopy. J. Microsc., 1998, 190(1-2), P. 45-51. 